\title{
Integrin $\alpha 7$ knockdown suppresses cell proliferation, migration, invasion and EMT in hepatocellular carcinoma
}

\author{
ZHIYONG WU* ${ }^{*}$ XIAOYU KONG ${ }^{*}$ and ZHIHUI WANG \\ Department of General Surgery, The Central Hospital of Wuhan, Tongji Medical College, \\ Huazhong University of Science and Technology, Wuhan, Hubei 430014, P.R. China
}

Received October 12, 2019; Accepted September 18, 2020

DOI: $10.3892 / e t m .2021 .9740$

\begin{abstract}
The present study aimed to investigate the effects of integrin $\alpha 7$ (ITGA7) on regulating hepatocellular carcinoma (HCC) progression and endothelial-mesenchymal transition (EMT). ITGA7 mRNA and protein expression in human normal liver epithelial cells and HCC cell lines were determined by reverse transcription-quantitative PCR (RT-qPCR) and western blotting. ITGA7 small interfering RNA [siRNA; ITGA7-knockdown (KD) group] and nonsense siRNA (control group) were transfected into Huh7 cells and SNU449 cells, respectively. ITGA7 mRNA and protein expression (RT-qPCR and western blotting, respectively), cell proliferation (Cell Counting Kit-8 assay), apoptosis (annexin V/propidium iodide assay), migration (wound scratch assay) and invasion (Transwell assay) were then detetected. E-cadherin, $\alpha$-smooth muscle actin ( $\alpha$-SMA), vimentin and $\mathrm{V}$-cadherin levels (RT-qPCR and western blotting) were also assessed. ITGA7 mRNA and protein expression levels were increased in Li7, Huh7, SKHEP1 and SNU449 cells compared with THLE-3 cells. Following transfection, ITGA7 mRNA and protein expression was lower in the ITGA7-KD group compared with that in the control group in both Huh7 and SNU449 cells, indicating successful transfection. In the ITGA7-KD group, cell proliferation decreased at 48 and $72 \mathrm{~h}$, cell apoptosis rates increased at $48 \mathrm{~h}$, cell migration rate was reduced at $24 \mathrm{~h}$ and cell invasion decreased at $24 \mathrm{~h}$ compared with the control group. Additionally, increased E-cadherin but decreased $\alpha$-SMA, vimentin and V-cadherin mRNA and protein expression levels were observed in the ITGA7-KD group compared with the control group at $24 \mathrm{~h}$.
\end{abstract}

Correspondence to: Dr Zhihui Wang, Department of General Surgery, The Central Hospital of Wuhan, Tongji Medical College, Huazhong University of Science and Technology, 26 Nanjing Road, Jiang'an, Wuhan, Hubei 430014, P.R. China

E-mail: huixin84002@163.com

${ }^{*}$ Contributed equally

Key words: integrin $\alpha 7$, hepatocellular carcinoma, cell proliferation, apoptosis, endothelial-mesenchymal transition
In conclusion, ITGA7 knockdown suppressed HCC progression and inhibited EMT in HCC in vitro, implying that ITGA7 might be a novel treatment target for HCC.

\section{Introduction}

Liver cancer was the sixth most common cancer and the fourth leading cause of cancer deaths worldwide in 2018, which resulted in 841,080 new cases and 781,265 deaths, accounting for $4.7 \%$ of all cancer cases and $8.2 \%$ of all cancer deaths (1). As the most common type of liver cancer, hepatocellular carcinoma (HCC) comprises $75-85 \%$ of cases of liver cancer (1). Surgical resection is an optimal modality in patients with HCC who have small solitary tumors and well-preserved liver function, and could be subjected to this invasive operation and achieve satisfactory efficacy (2). Patients who are diagnosed in advanced stages of HCC, who account for the majority of all cases, are often not suitable candidates for surgical resection (2). Liver transplantation, another curative treatment option, is most suitable for patients with $\mathrm{HCC}$ who are not good candidates for resection, although donor shortage and high medical costs limit its application (2). Hence, investigating the molecular mechanisms of HCC progression is required for the detection of novel and effective therapeutic targets to improve HCC prognosis.

Integrins, which are heterodimers, consist of $\alpha$ and $\beta$ subunits, which participate in a range of cellular processes including cell survival, growth, invasion and migration (3). Integrin $\alpha 7$ (ITGA7), which belongs to the integrin family of adhesion molecules, plays a role in cell-cell and cell-extracellular matrix interactions in multiple cellular processes (4). According to previous studies, ITGA7 is oncogenic in the pathological processes of several carcinomas including glioblastoma, esophageal squamous cell carcinoma (OSCC) and lung cancer (4-6). For instance, ITGA7 promotes OSCC cell migration and invasion, while concurrently increasing E-cadherin and $\alpha$-smooth muscle actin ( $\alpha$-SMA) expression, which are common markers of endothelial-mesenchymal transition (EMT), indicating that ITGA7 may promote malignant cellular function and induce EMT in OSCC cells (5). Hence, it was hypothesized that ITGA7 may also have promotive effects on cellular function and EMT regulation in HCC. However, to the best of our knowledge, little is known about the role of ITGA7 in HCC. Thus, the present study aimed to 
investigate the effects of ITGA7 on regulating HCC progression and EMT.

\section{Materials and methods}

Cell culture. Human normal liver epithelial cells (THLE-3) and HCC cell lines SKHEP1 and SNU449 were purchased from American Type Culture Collection. HCC cell lines Li7 and Huh7 were purchased from RIKEN BioResource Center. THLE-3 cells were cultured in 90\% bronchial epithelial growth medium (Lonza Group, Ltd.) and 10\% FBS (Gibco; Thermo Fisher Scientific, Inc.). Li7 cells and SNU449 cells were cultured in 90\% RPMI-1640 medium (Gibco; Thermo Fisher Scientific, Inc.) and 10\% FBS. Huh7 cells were cultured in 90\% DMEM (Gibco; Thermo Fisher Scientific, Inc.) and 10\% FBS. SKHEP1 cells were cultured in 90\% Eagle's minimum essential medium (Gibco; Thermo Fisher Scientific, Inc.) and 10\% FBS. All medium was added $100 \mathrm{U} / \mathrm{ml}$ penicillin and $100 \mathrm{lg} / \mathrm{ml}$ treptomycin (Sigma-Aldrich; Merck KGaA). Cells were maintained in a humid incubator at $37^{\circ} \mathrm{C}$.

ITGA7 small interfering RNA (siRNA) construction and transfection. siRNA was used to knock down ITGA7 expression. ITGA7 siRNA and nonsense siRNA were designed and synthesized by Guangzhou RiboBio Co., Ltd. ITGA7 siRNA $(80 \mathrm{nM})$ and nonsense siRNA were transfected into Huh7 cells and SNU449 cells using Lipofectamine ${ }^{\circledR} 2000$ (Invitrogen; Thermo Fisher Scientific, Inc.) according to the manufacturer's instructions for $6 \mathrm{~h}$ at $37^{\circ} \mathrm{C}$. Subsequently, cells transfected with ITAG7 siRNA were considered ITGA7-knockdown (KD) cells, and cells transfected with nonsense siRNA were marked as control cells. At $24 \mathrm{~h}$ after transfection, ITGA7 mRNA and protein expression levels were determined by RT-qPCR and western blotting; cell apoptosis was detected by an annexin $\mathrm{V} /$ propidium iodide (AV/PI) assay at $48 \mathrm{~h}$, expression of apoptosis-related protein cleaved caspase 3 was detected by western blotting, and cell migration and invasion abilities were assessed by wound scratch and Transwell assays, respectively. Cell viability was assessed using a Cell Counting Kit-8 (CCK-8) assay at 0, 24, 48 and $72 \mathrm{~h}$. Additionally, whether the mRNA and protein expression of E-cadherin, vimentin, $\mathrm{N}$-cadherin and $\alpha$-SMA were regulated by ITGA7 was determined by RT-qPCR and western blotting at $24 \mathrm{~h}$ after transfection. In addition, the sequences of ITGA7 siRNA were as follows: Forward, 5'-GCAUCAAGAGCUUCGGCUATT-3' and reverse, 5'-UAGCCGAAGCUCUUGAUGCTT-3'.

$R T-q P C R$. ITGA7 mRNA expression was assessed in THLE-3, Li7, Huh7, SKHEP1 and SNU449 cells. E-cadherin, vimentin, $\mathrm{N}$-cadherin and $\alpha$-SMA mRNA expression was assessed in Huh7 and SNU449 cells. Following cell dissociation using $0.25 \%$ trypsin (Gibco; Thermo Fisher Scientific, Inc.), TRIzol ${ }^{\circledR}$ reagent (Invitrogen; Thermo Fisher Scientific, Inc.) was used to extract total RNA. Subsequently, $1 \mu \mathrm{g}$ RNA was reverse transcribed into cDNA using a PrimeScript RT reagent kit (Takara Bio, Inc.) with following thermocycling condition: $42^{\circ} \mathrm{C}$ for $15 \mathrm{~min}$ and $85^{\circ} \mathrm{C}$ for $5 \mathrm{sec}$. TB Green ${ }^{\mathrm{TM}}$ Fast qPCR mix (Takara, Bio, Inc.) was used for qPCR. The following thermocycling conditions were used: $95^{\circ} \mathrm{C}$ for $5 \mathrm{~min}$; 40 cycles of $95^{\circ} \mathrm{C}$ for $5 \mathrm{sec}$ and $61^{\circ} \mathrm{C}$ for $30 \mathrm{sec}$. Gene expression was calculated using the $2^{-\Delta \Delta C q}$ method (7). Primer sequences are shown in Table I. GAPDH was used as the internal reference gene. In addition, the sequences of ITGA7 siRNA were as follows: Forward, 5'-GCAUCAAGAGCUUCGGCUATT-3' and reverse, 5'-UAGCCGAAGCUCUUGAUGCTT-3'.

Western blotting. Total protein was extracted using RIPA Lysis and Extraction buffer (Thermo Fisher Scientific, Inc.). The Pierce $^{\mathrm{TM}}$ BCA Protein Assay kit (Thermo Fisher Scientific, Inc.) was used to measure protein concentration. Subsequently, $20 \mu \mathrm{g}$ protein sample was fractionated using NuPAGE ${ }^{\mathrm{TM}}$ 4-20\% Tris-Acetate Midi Protein Gels (Thermo Fisher Scientific, Inc.), and transferred to PVDF membranes. Membranes were blocked using 5\% skim milk for $2 \mathrm{~h}$ at room temperature and incubated with primary antibodies overnight at $4^{\circ} \mathrm{C}$. Membranes were incubated with a secondary antibody for $1 \mathrm{~h}$ at room temperature. The chemiluminescence of blots was detected using Pierce ${ }^{\mathrm{TM}}$ ECL Plus Western Blotting substrate (Invitrogen; Thermo Fisher Scientific, Inc.) and then exposed to X-ray film (Kodak) following treatment. GAPDH was used as the internal reference protein. Antibodies used for western blotting are listed in Table II.

CCK-8 assay. Cells were plated at a density of $3 \times 10^{4}$ the 96-well plates for $24 \mathrm{~h}$. Following the addition of $10 \mu \mathrm{l} \mathrm{CCK-8}$ solution (Dojindo Molecular Technologies, Inc.) and $90 \mu \mathrm{l}$ RPMI-1640 medium to each plate, cells were incubated at $37^{\circ} \mathrm{C}$ with $5 \% \mathrm{CO}_{2}$. Optical density values were detected using a microplate reader (Biotek Instruments, Inc.).

$A V / P I$. Cells were digested with pancreatin and washed with PBS. Following suspension in $100 \mu 1$ binding buffer and addition of $5 \mu \mathrm{l} \mathrm{AV}$ and $5 \mu \mathrm{l} \mathrm{PI}$, cells were incubated in the dark with a Annexin V-FITC Apoptosis Detection kit according to the manufacturer's protocol (Sigma-Aldrich; Merck KGaA).

Wound scratch assay. Cells, which were pre-culture in medium containing $1 \%$ FBS for $24 \mathrm{~h}$, were cultured until $80 \%$ confluence and scraped with a sterile pipette tip to create adherent cell gaps. Subsequently, the cells were incubated and then observed at 0 and $24 \mathrm{~h}$ by inverted fluorescence microscopy (Nikon Corporation). The migration rate was calculated as follows: Migration rate $=($ scraped area- residual area $) /$ scraped area.

Transwell assay. After coating Matrigel basement membrane matrix (BD Biosciences) on the upper Transwell chamber (Costar; Corning, Inc) at $37^{\circ} \mathrm{C}$ for $1 \mathrm{~h}$. Cells $\left(3 \times 10^{4}\right)$ in FBS-free medium (DMEM for Huh-7 cells and RPMI-1640 for SNU-449 cells) were seeded in the upper chamber, and lower chamer was filled with $500 \mu 1$ 10\% FBS containng-medim (DMEM for Huh-7 cells and RPMI-1640 for SNU-449 cells). Following incubation for $24 \mathrm{~h}$ at $37^{\circ} \mathrm{C}$ and wiping of the upper cells, cells in the lower chamber were fixed with formaldehyde (Sigma-Aldrich; Merck KGaA). After staining with $0.5 \%$ crystal violet (Sigma-Aldrich; Merck KGaA) for $15 \mathrm{~min}$ at room temperature, the invasive cell count of each well was calculated by the averaging the invasive cell count 
Table I. Primers used for reverse transcription-quantitative PCR.

Primer sequence (5'-3')

\begin{tabular}{lll} 
Target gene & \multicolumn{1}{c}{ Forward } & \multicolumn{1}{c}{ Reverse } \\
\hline ITGA7 & GCCACTCTGCCTGTCCAATG & GGAGGTGCTAAGGATGAGGTAGA \\
E-cadherin & TGATTCTGCTGCTCTTGCTGTT & CCTCTTCTCCGCCTCCTTCTT \\
$\alpha-S M A$ & CATTCACGAGACCACCTACAACAG & CGCCGATCCACACCGAGTAT \\
GAPDH & GACCACAGTCCATGCCATCAC & ACGCCTGCTTCACCACCTT
\end{tabular}

ITGA7, integrin $\alpha 7 ; \alpha$-SMA, $\alpha$-smooth muscle actin.

Table II. Antibodies used for western blotting.

\begin{tabular}{lll}
\hline Antibody & \multicolumn{1}{c}{ Manufacturer } & Catalog number \\
\hline Primary antibodies & & Dilution \\
ITGA7 mouse mAb & Santa Cruz Biotechnology, Inc. & sc-51576 \\
E-cadherin mouse mAb & Santa Cruz Biotechnology, Inc. & sc-8426 \\
Vimentin mouse mAb & Santa Cruz Biotechnology, Inc. & sc-6260 \\
N-cadherin mouse mAb & Santa Cruz Biotechnology, Inc. & sc-393933 \\
$\alpha-S M A$ mouse mAb & Santa Cruz Biotechnology, Inc. & sc-53142 \\
Cleaved caspase 3 mouse mAb & Cell Signaling Technology, Inc. & $9664 S$ \\
GAPDH mouse mAb & Santa Cruz Biotechnology, Inc. & sc-47724 \\
Secondary antibodies & & $1: 1,000$ \\
Goat anti-mouse IgG-HRP & Santa Cruz Biotechnology, Inc. & $1: 1,000$ \\
\hline
\end{tabular}

ITGA7, integrin $\alpha 7 ; \alpha$-SMA, $\alpha$-smooth muscle actin.

of five fields of view in each well, which was observed using an inverted fluorescence microscopy (Nikon Corporation) at a magnification of $\mathrm{x} 200$.

Statistical analysis. Statistical analysis and graph plotting were performed using GraphPad Prism 7.02 (GraphPad Software, Inc.). All assays were repeated in triplicate. Data are presented as the mean $\pm \mathrm{SD}$. Comparison between two groups were performed using unpaired t-test, while multiple comparisons were performed using Dunnett's t-test. $\mathrm{P}<0.05$ was considered to indicate a statistically significant difference.

\section{Results}

ITGA7 is highly expressed in HCC cell lines compared with human normal liver epithelial cells. ITGA7 mRNA expression was higher in HCC cell lines [including Li7 $(\mathrm{P}<0.01)$, Huh7 $(\mathrm{P}<0.001)$, SKHEP1 $(\mathrm{P}<0.01)$ and SNU449 $(\mathrm{P}<0.05)$ cells] compared with human normal liver epithelial cells (THLE-3 cells) (Fig. 1A). In addition, ITGA7 protein expression was elevated in HCC cell lines [Li7 $(\mathrm{P}<0.01)$, Huh7 $(\mathrm{P}<0.001)$, SKHEP1 $(\mathrm{P}<0.001)$ and SNU449 $(\mathrm{P}<0.05)$ cells] compared with human normal liver epithelial cells (THLE-3 cells; Fig. 1B and C). ITGA7 expression was the highest in
Huh7 cells and the lowest in SNU449 cells. Hence, Huh7 and SNU449 cells were selected for subsequent experiments to assess the effects of ITGA7 knockdown on cell proliferation, migration, invasion and EMT in HCC cells.

ITGA7 expression is attenuated in the ITGA7-KD group compared with the control group after transfection. ITGA7 mRNA ( $\mathrm{P}<0.001$; Fig. 2A) and protein (Fig. 2C) expression decreased in the ITGA7-KD group compared with the control group in Huh7 cells. ITGA7 mRNA ( $<<0.001$; Fig. 2B) and protein (Fig. 2D) expression levels were also lower in the ITGA7-KD group compared with the control group in SNU449 cells.

ITGA7 knockdown decreases cell proliferation but increases cell apoptosis. The effects of ITGA7 on regulating cell proliferation and apoptosis in Huh7 and SNU449 cells were then investigated. In Huh7 cells, cell proliferation decreased in the ITGA7-KD group at $48 \mathrm{~h}(\mathrm{P}<0.05)$ and $72 \mathrm{~h}(\mathrm{P}<0.01)$ compared with the control group (Fig. 3A). Meanwhile, the cell apoptosis rate at $48 \mathrm{~h}$ was increased in the ITGA7-KD group compared with the control group $(\mathrm{P}<0.01$; Fig. $3 \mathrm{C}$ and $\mathrm{E})$, and cleaved caspase 3 protein expression was increased in the ITGA7-KD group compared with the control group (Fig. 3G). In SNU449 cells, cell proliferation was reduced 

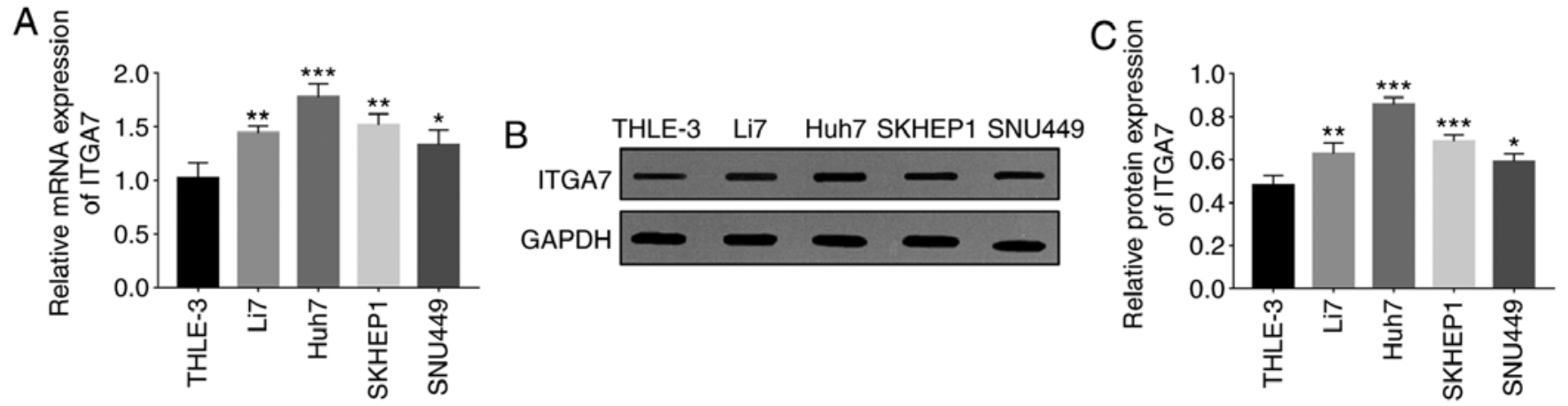

Figure 1. ITGA7 expression levels in cell lines. (A) ITGA7 mRNA expression in HCC cell lines and human normal liver epithelial cell line. (B and C) ITGA7 protein expression in HCC cell lines and human normal liver epithelial cell line. ITGA7, integrin $\alpha 7$; HCC, hepatocellular carcinoma. ${ }^{*} \mathrm{P}<0.05,{ }^{* *} \mathrm{P}<0.01$ and **** $\mathrm{P}<0.001$ vs. THLE-3 cells.
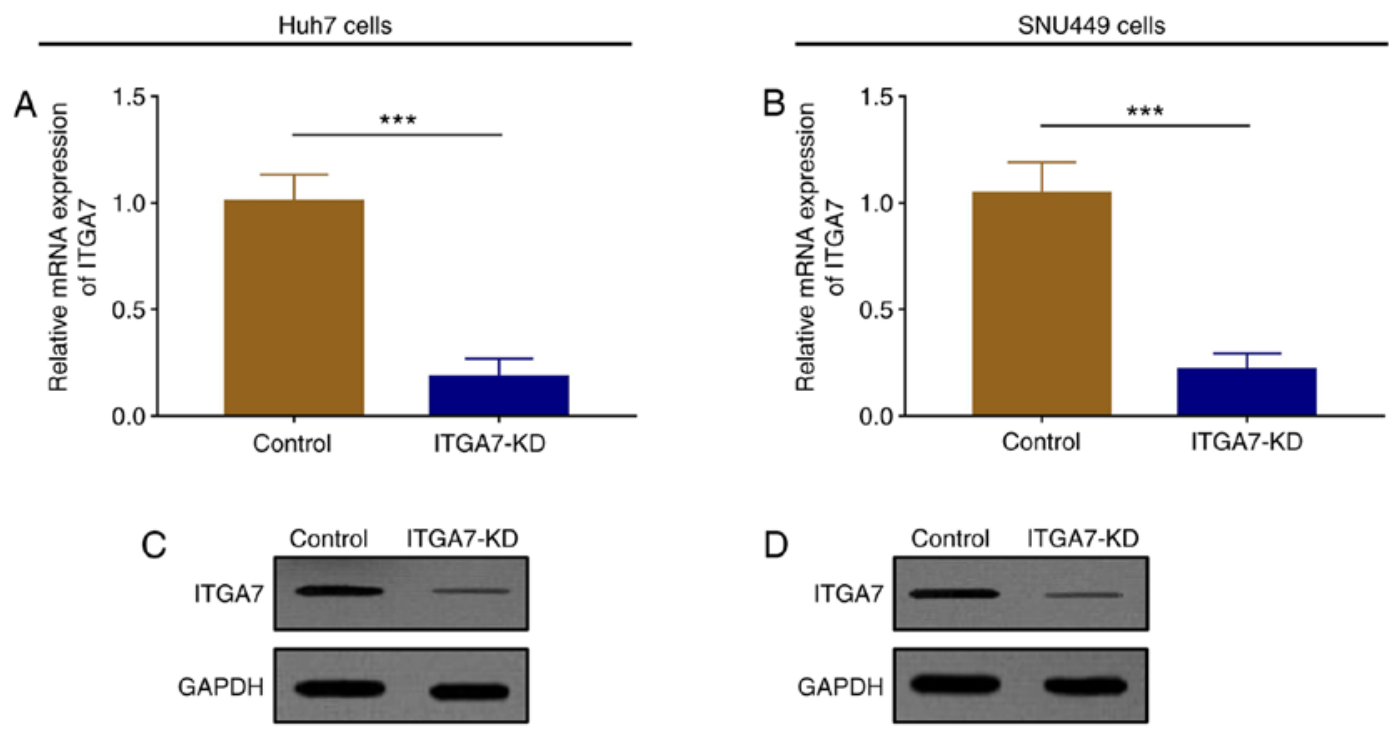

Figure 2. ITGA7 expression following transfection with ITGA7 small interfering RNA. ITGA7 mRNA expression in (A) Huh7 and (B) SNU449 cells. ITGA7 protein expression in (C) Huh7 and (D) SNU449 cells. ITGA7, integrin $\alpha 7$; KD, knockdown. ${ }^{* * *} \mathrm{P}<0.001$.

in the ITGA7-KD group at $48 \mathrm{~h}(\mathrm{P}<0.05)$ and $72 \mathrm{~h}(\mathrm{P}<0.05)$ compared with the control group (Fig. 3B). The cell apoptosis rate at $48 \mathrm{~h}$ was enhanced in the ITGA7-KD group compared with the control group $(\mathrm{P}<0.01$; Fig. 3D and $\mathrm{F})$, and cleaved caspase 3 protein expression increased in the ITGA7-KD group compared with the control group (Fig. 3H).

ITGA7 knockdown represses cell migration. The effects of ITGA7 on regulating cell migration in Huh7 and SNU449 cells were assessed. In Huh7 cells, the wound scratch assay showed that the migration rate at $24 \mathrm{~h}$ after transfection was lower in the ITGA7-KD group compared with the control group ( $\mathrm{P}<0.01$; Fig. $4 \mathrm{~A}$ and $\mathrm{C})$. In SNU449 cells, the migration rate at $24 \mathrm{~h}$ after transfection was also attenuated in the ITGA7-KD group compared with the control group $(\mathrm{P}<0.001$; Fig. 4B and D).

ITGA7 knockdown suppresses cell invasion. Transwell assays were performed to investigate the effects of ITGA7 on regulating Huh7 and SNU449 cell invasion. The number of invasive Huh7 cells was decreased in the ITGA7-KD group compared with the control group $(\mathrm{P}<0.01)$ at $24 \mathrm{~h}(\mathrm{Fig} .5 \mathrm{~A}$ and $\mathrm{C})$. The number of invasive SNU449 cells was also lower in the ITGA7-KD group compared with the control group $(\mathrm{P}<0.01)$ at $24 \mathrm{~h}$ (Fig. 5B and D).

ITGA7 knockdown represses EMT. To assess the effects of ITGA7 on EMT underlying HCC pathogenesis, the expression levels of EMT markers, including E-cadherin, vimentin, $\mathrm{N}$-cadherin and $\alpha$-SMA, were detected in Huh7 and SNU449 cells after transfection. In Huh7 cells, mRNA $(\mathrm{P}<0.05)$ and protein expression of $\mathrm{E}$-cadherin increased (Fig. 6A and I); however, mRNA (all $\mathrm{P}<0.05$ ) and protein expression levels of vimentin (Fig. 6C and I), N-cadherin (Fig. 6E and I) and $\alpha$-SMA (Fig. 6G and I) were decreased in the ITGA7-KD group compared with the control group at $24 \mathrm{~h}$. In SNU449 cells, mRNA $(\mathrm{P}<0.01)$ and protein expression levels of E-cadherin (Fig. 6B and J) increased, while mRNA (all $\mathrm{P}<0.05$ ) and protein expression levels of vimentin (Fig. 6D and J), N-cadherin (Fig. 6F and J) and $\alpha$-SMA (Fig. $6 \mathrm{H}$ and $\mathrm{J}$ ) were decreased in the ITGA7-KD group compared with the control group at $24 \mathrm{~h}$. 
Huh7 cells
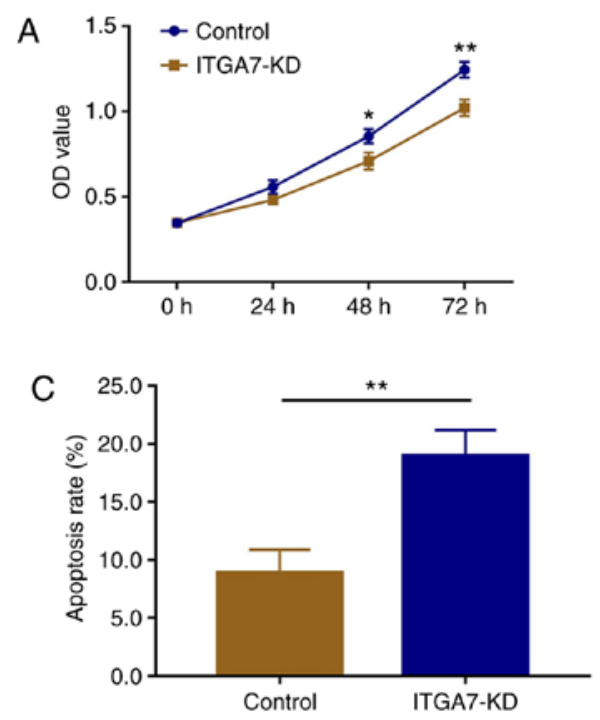

E
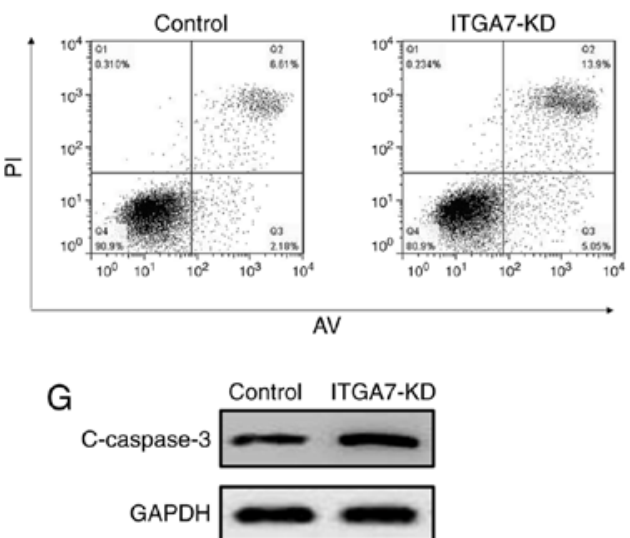

SNU449 cells
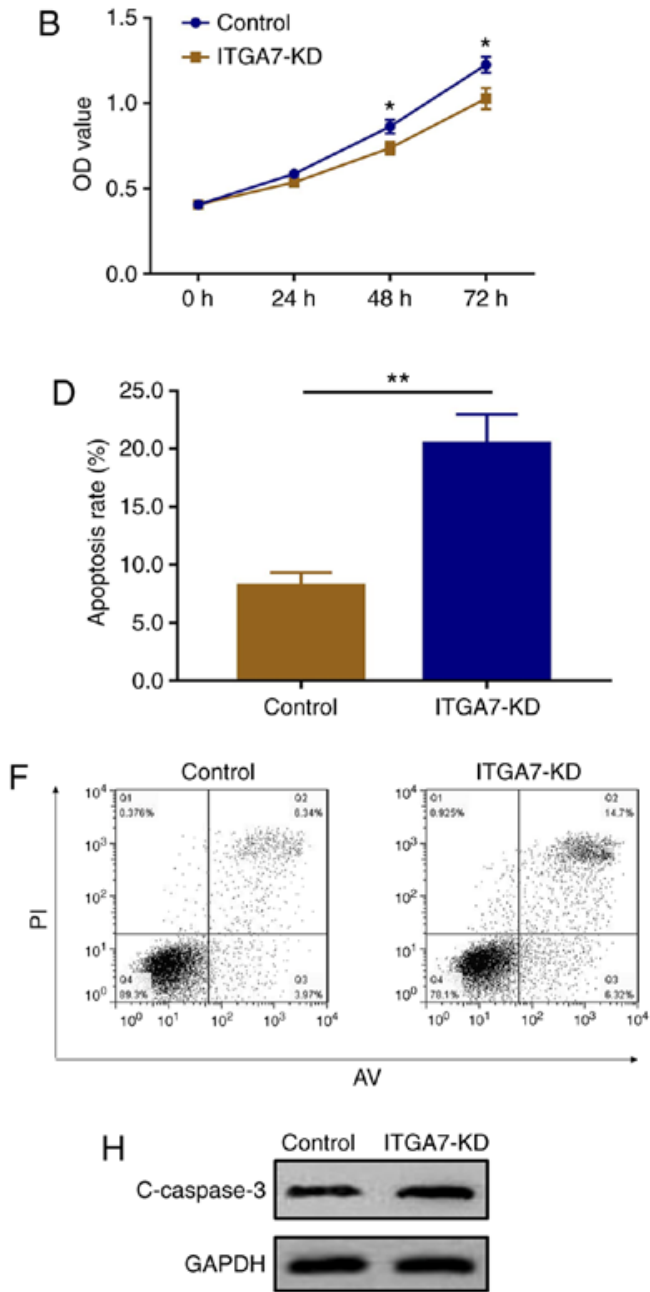

Figure 3. Cell proliferation and apoptosis. Proliferation of (A) Huh7 and (B) SNU449 cells following transfection. ${ }^{*} \mathrm{P}<0.05$ and ${ }^{* *} \mathrm{P}<0.01$ vs. the control group. Apoptosis rate of (C) Huh7 and (D) SNU449 cells following transfection. ${ }^{* *} \mathrm{P}<0.01$. Representative flow cytometry plots used to determine apoptosis rates in (E) Huh7 and (F) SNU449 cells. Cleaved caspase 3 and GAPDH protein expression following transfection in (G) Huh7 and (H) SNU449 cells. ITGA7, integrin $\alpha 7$; C-caspase 3; cleaved caspase 3; KD, knockdown; AV, annexin V; PI, propidium iodide.
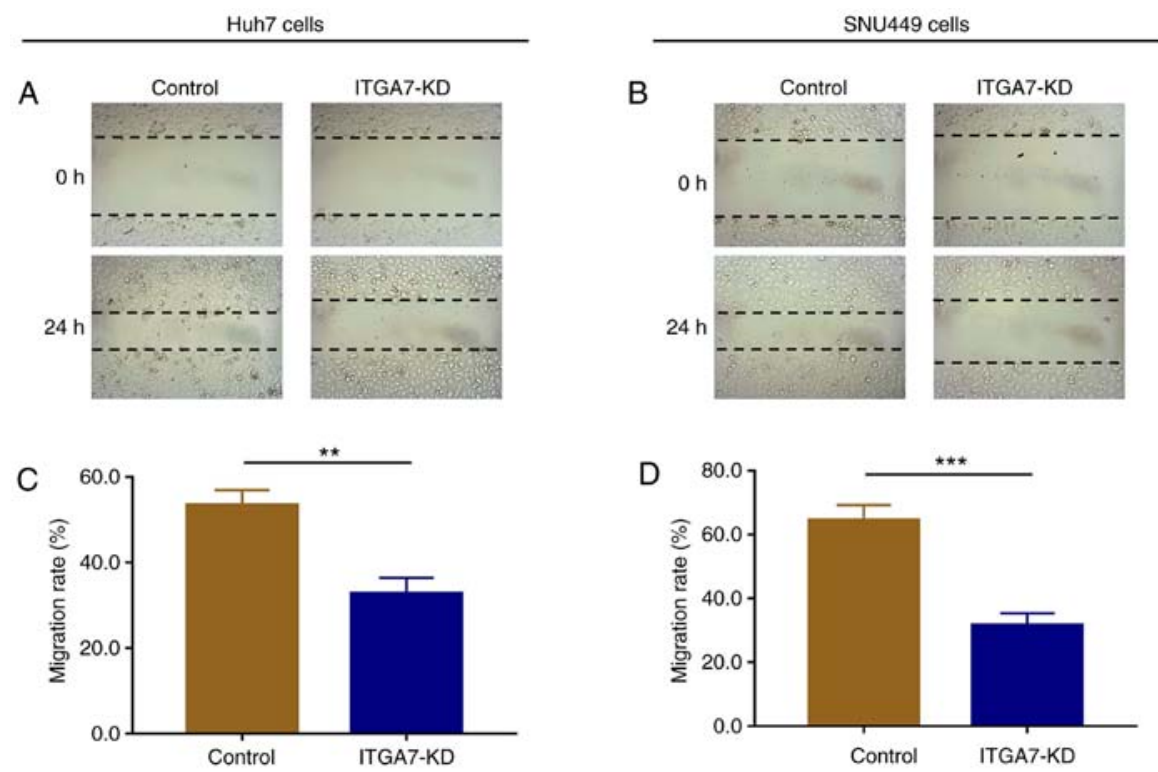

Figure 4. Cell migration. Representative wound scratch assay images of (A) Huh7 and (B) SNU44 cells. (C) Huh7 and (D) SNU44 cell migration rates. Magnification, $\mathrm{x} 200 .{ }^{* *} \mathrm{P}<0.01$ and ${ }^{* * *} \mathrm{P}<0.001$. ITGA7, integrin $\alpha 7 ; \mathrm{KD}$, knockdown. 


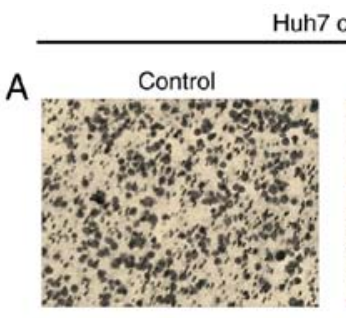

Huh7 cells

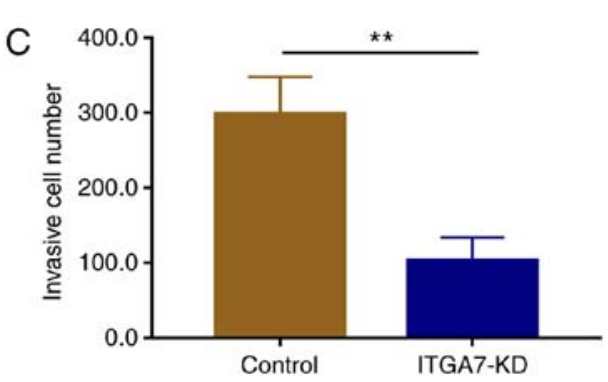

ITGA7-KD

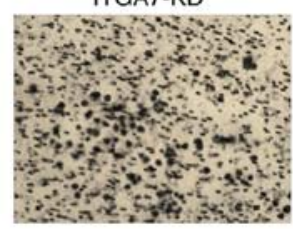

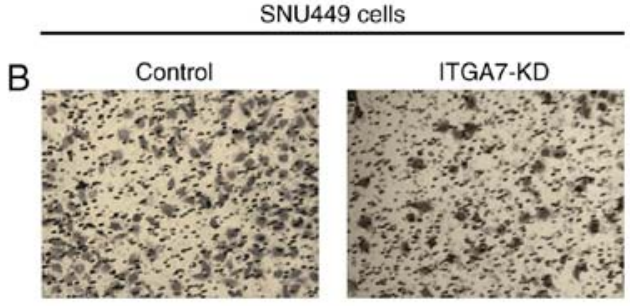

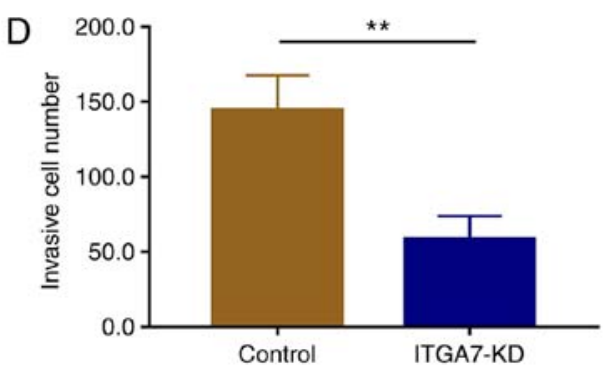

Figure 5. Cell invasion. Representative Transwell assay images of (A) Huh7 and (B) SNU44 cells. (C) Huh7 and (D) SNU44 cell invasion. Magnification, x200. ${ }^{* *} \mathrm{P}<0.01$. ITGA7, integrin $\alpha 7 ; \mathrm{KD}$, knockdown.

\section{Discussion}

Integrins are transmembrane protein receptors that attach cells to the extracellular matrix and bind ligands secreted by other cells $(8,9)$. As one of the integrins, ITGA7 is proposed to serve as a key regulator in tumor propagation and cancer stem cell properties $(5,10)$. Previous studies revealed that ITGA7 is highly expressed in various cancer cells, including OSCC and mesothelioma $(5,11)$. Although these previous studies detected an upregulation of ITGA7 in various cancer cells, to the best of our knowledge, its role in HCC is still unclear. The present study revealed that ITGA7 was overexpressed in HCC cell lines, including Li7, Huh7, SKHEP1 and SNU449 cells, compared with human normal liver epithelial THLE-3 cells. ITGA7 regulates several genes and pathways, including the focal adhesion kinase (FAK)/AKT-zinc finger E-box Binding Homeobox 1 (ZEB1) signaling pathway, to promote cell proliferation and metastasis, subsequently contributing to the malignant transformation of $\operatorname{HCC}(5,12)$. Thus, ITGA7 was overexpressed in HCC cells compared with healthy control cells.

Previous studies indicated that ITGA7 is involved in the pathological progression of different carcinomas through affecting cell activities such as cell migration and invasion (5,6,9,13-15). For example, the interaction between ITGA7 and laminin-induced outside-in signaling contributed to glioblastoma stem-like cell growth and invasion (12). Furthermore, the interaction between ITGA7 and S100P activated the FAK/AKT-ZEB1 signaling pathway, which induced lung cancer cell migration and invasion (6). Furthermore, ITGA7 is associated with cancer cell stemness. In another study, ITGA7 promoted the stemness of OSCC cells via FAK/MAPK/ERK signaling, which subsequently induced the tumorigenicity and metastasis of OSCC (5). In addition, ITGB7 knockdown enhanced cell apoptosis but inhibited cell proliferation and invasion in breast cancer (14). Although a few studies have been performed to explore the role of ITGA7 in different types of carcinoma, there remain certain contradictions. Several lines of evidence revealed the role of ITGA7 as a tumor suppressor in various malignancies. For example, ITGA7 appears to activate cyclin-dependent kinase inhibitor 3 (CDKN3) and Rac GTPase-activating protein 1 (RACGAP1) expression to inhibit cell motility and metastasis of HCC cells (16). Another study revealed that ITGA7 may be a tumor suppressor that impedes tumor growth and inhibits migration in prostate cancer (9). Additionally, ITGB7 interacts with high temperature requirement $\mathrm{A} 2$ to promote prostate cancer cell death (13). To the best of our knowledge, little is known about the role of ITGA7 in HCC. The present study investigated the effect of ITGA7 on regulating HCC cell activities. It was found that ITGA7 knockdown decreased cell proliferation, migration and invasion, but increased apoptosis of HCC cells, which suggested that ITGA7 knockdown might suppress the function of HCC cells. There are a few possible explanations for this. Similar to its cancerogenic effect on tumor progression in lung cancer, ITGA7 might interact with S100P to trigger FAK/AKT-ZEB1 signaling to enhance HCC cell proliferation, migration and invasion, thereby contributing to HCC tumor progression (6). Similar to the promotive effects of ITGA7 on tumor progression in glioblastoma, ITGA7 might accelerate HCC cell growth and invasion via interacting with laminin-induced outside-in signaling, thereby leading to tumor progression of HCC (12). ITGA7 may attach cells to the extracellular matrix and interact with ligands secreted by other cells to activate HCC cell invasion and migration, which subsequently promotes tumor progression of $\operatorname{HCC}(8,9)$. ITGA7 may also regulate CDKN3, which dephosphorylates tyrosine residues of different cyclin-dependent kinases and represses cell cycle progression in yeast and mammalian cells, to increase HCC cell invasion and motility, subsequently accelerating tumor progression in HCC (16-18). ITGA7 also may modulate RACGAP1 to increase cell growth, enhance cell motility and promote tumor metastasis. Taken together, it may be hypothesized that ITGA7 knockdown suppresses tumor progression of HCC $(16,19)$. In addition, the discrepancies in results between the present study and previous studies 

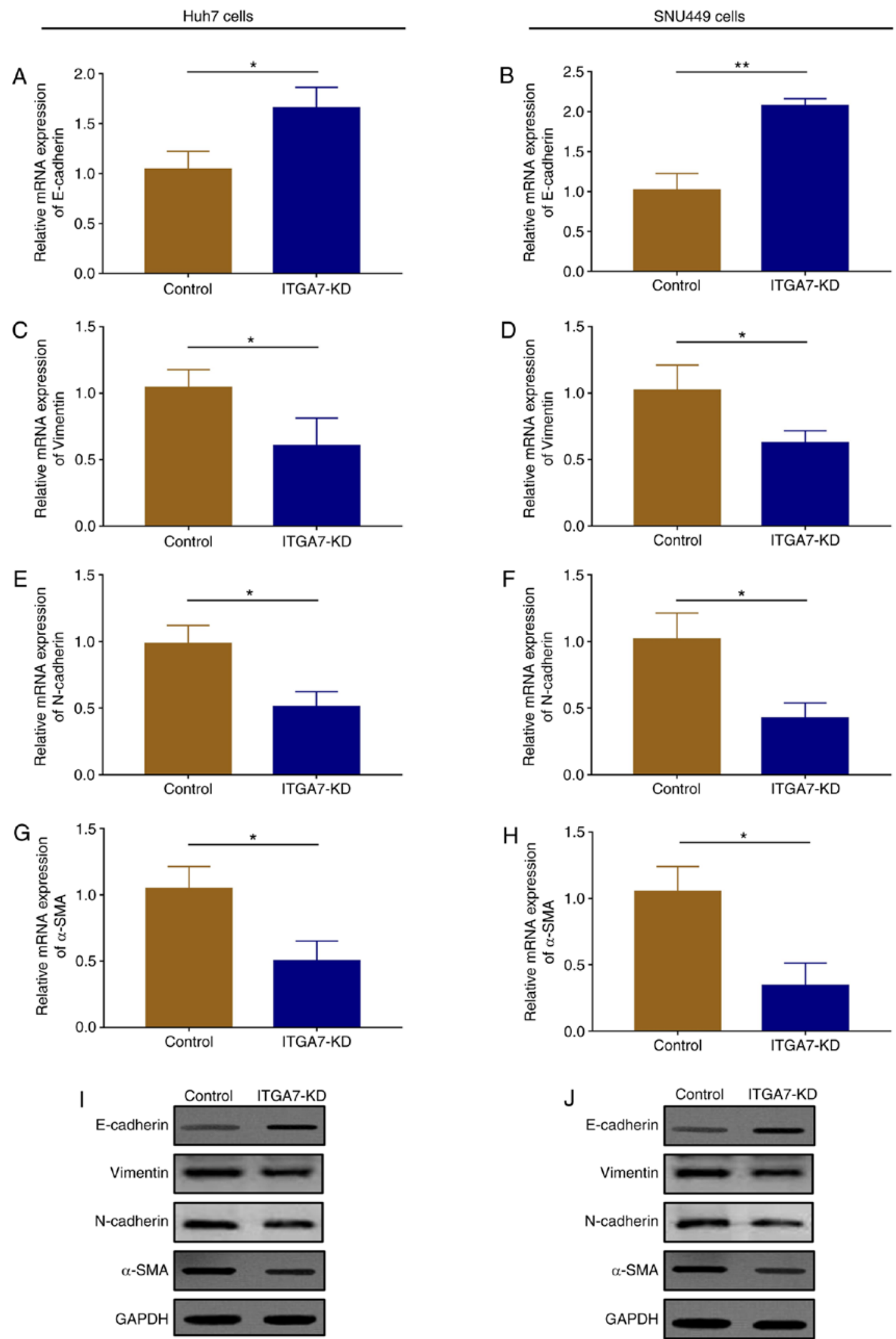

Figure 6. Effects of ITGA7 on endothelial-mesenchymal transition. (A and B) E-cadherin, (C and D) vimentin, (E and F) N-cadherin and (G and H) $\alpha$-SMA mRNA expression after transfection in Huh7 cells and SNU449 cells. Protein expression of E-cadherin, vimentin, N-cadherin and $\alpha$-SMA after transfection in (J) Huh7 and (I) SNU449 cells. "P<0.05 and ${ }^{* *} \mathrm{P}<0.01$. ITGA7, integrin $\alpha 7$; $\alpha$-SMA, $\alpha$-smooth muscle actin; KD, knockdown.

might result from differences between the malignances studied. The present study focused on HCC, while the majority of previous studies focused on other types of cancer. Due to the complexity of malignant pathological processes, different malignances might be distinctive in terms of pathological features. Thus, the effects of ITGA7 on cellular function and 
its underlying mechanisms in other malignances may differ. Besides, different cell lines, different assay operation times and experimental procedures may have also contributed to distinctive results among different studies.

To the best of our knowledge, limited information is available regarding the role of ITGA7 in EMT and tumor metastasis $(5,20,21)$. One previous study reported that ITGA7 promoted OSCC cell migration and invasion and induced EMT (5). EMT is not only a well-coordinated process controlled by multiple signaling pathways during embryonic development, but also a pathological characteristic in neoplasia and fibrosis (22-25). EMT has been considered as an essential regulator linked to tumor progression and tumor metastasis through accelerating cancer cell invasion and dissemination to distant organs (22-25). To assess the effects of ITGA7 on regulating EMT in HCC, E-cadherin, $\alpha$-SMA, vimentin and $\mathrm{N}$-cadherin levels were detected. ITGA7 knockdown increased E-cadherin expression and decreased $\alpha$-SMA expression in HCC cells. To summarize, ITGA7 knockdown may repress EMT in HCC. However, how ITGA7 knockdown suppressed cell proliferation, migration, invasion and ETM in HCC remains unclear. Further experiments, such as RNA sequencing, bioinformatics and subsequent validation by RT-qPCR are required.

In conclusion, ITGA7 knockdown suppressed HCC cell proliferation, migration, invasion and EMT, and promoted apoptosis. These data indicated that ITGA7 might be a novel and effective treatment target for HCC.

\section{Acknowledgements}

Not applicable.

\section{Funding}

No funding was received.

\section{Availability of data and materials}

The datasets used and/or analyzed during the current study are available from the corresponding author on reasonable request.

\section{Authors' contributions}

$\mathrm{ZWu}$ and $\mathrm{XK}$ made substantial contributions to the design of the present study, ZWu, XK and ZW were responsible for data acquisition and interpretation. All authors read and approved the final manuscript. All authors agree to be accountable for all aspects of the work in ensuring that questions related to the accuracy or integrity of the work are appropriately investigated and resolved.

\section{Ethics approval and consent to participate}

Not applicable.

\section{Patient consent for publication}

Not applicable.

\section{Competing interests}

The authors declare that they have no competing interests.

\section{References}

1. Bray F, Ferlay J, Soerjomataram I, Siegel RL, Torre LA and Jemal A: Global cancer statistics 2018: GLOBOCAN estimates of incidence and mortality worldwide for 36 cancers in 185 countries. CA Cancer J Clin 68: 394-424, 2018.

2. Forner A, Llovet JM and Bruix J: Hepatocellular carcinoma. Lancet 379: 1245-1255, 2012.

3. Desgrosellier JS and Cheresh DA: Integrins in cancer: Biological implications and therapeutic opportunities. Nat Rev Cancer 10: 9-22, 2010.

4. Carrasco-Garcia E, Auzmendi-Iriarte J and Matheu A: Integrin $\alpha 7$ : A novel promising target in glioblastoma stem cells. Stem Cell Investig 5: 2, 2018.

5. Ming XY, Fu L, Zhang LY, Qin YR, Cao TT, Chan KW, Ma S, Xie D and Guan XY: Integrin $\alpha 7$ is a functional cancer stem cell surface marker in oesophageal squamous cell carcinoma. Nat Commun 7: 13568, 2016.

6. Hsu YL, Hung JY, Liang YY, Lin YS, Tsai MJ, Chou SH, Lu CY and Kuo PL: S100P interacts with integrin $\alpha 7$ and increases cancer cell migration and invasion in lung cancer. Oncotarget 6: 29585-29598, 2015.

7. Livak KJ and Schmittgen TD: Analysis of relative gene expression data using real-time quantitative PCR and the 2(-Delta Delta C(T)) Method. Methods 25: 402-408, 2001.

8. Hynes RO: Integrins: Bidirectional, allosteric signaling machines. Cell 110: 673-687, 2002.

9. Tan LZ, Song Y, Nelson J, Yu YP and Luo JH: Integrin $\alpha 7$ binds tissue inhibitor of metalloproteinase 3 to suppress growth of prostate cancer cells. Am J Pathol 183: 831-840, 2013.

10. Nunes AM, Barraza-Flores P, Smith CR and Burkin DJ: Integrin $\alpha 7$ : A major driver and therapeutic target for glioblastoma malignancy. Stem Cell Investig 4: 97, 2017.

11. Burkin DJ and Fontelonga TM: Mesothelioma cells breaking bad: Loss of integrin $\alpha 7$ promotes cell motility and poor clinical outcomes in patients. J Pathol 237: 282-284, 2015.

12. Haas TL, Sciuto MR, Brunetto L, Valvo C, Signore M, Fiori ME, di Martino S, Giannetti S, Morgante L, Boe A, et al: Integrin $\alpha 7$ Is a Functional Marker and Potential Therapeutic Target in Glioblastoma. Cell Stem Cell 21: 35-50.e9, 2017.

13. Zhu ZH, Yu YP, Zheng ZL, Song Y, Xiang GS, Nelson J, Michalopoulos $\mathrm{G}$ and Luo JH: Integrin alpha 7 interacts with high temperature requirement A2 (HtrA2) to induce prostate cancer cell death. Am J Pathol 177: 1176-1186, 2010.

14. Bai X, Gao C, Zhang L and Yang S: Integrin $\alpha 7$ high expression correlates with deteriorative tumor features and worse overall survival, and its knockdown inhibits cell proliferation and invasion but increases apoptosis in breast cancer. J Clin Lab Anal 33: e22979, 2019.

15. Su Y, Guan XQ, Liu FQ and Wang YL: The effects of MIBG on the invasive properties of HepG2 hepatocellular carcinoma cells. Int J Mol Med 34: 842-848, 2014.

16. Ren B, Yu YP, Tseng GC, Wu C, Chen K, Rao UN, Nelson J, Michalopoulos GK and Luo JH: Analysis of integrin alpha7 mutations in prostate cancer, liver cancer, glioblastoma multiforme, and leiomyosarcoma. J Natl Cancer Inst 99: 868-880, 2007.

17. Gyuris J, Golemis E, Chertkov H and Brent R: Cdi1, a human G1 and S phase protein phosphatase that associates with Cdk2. Cell 75: 791-803, 1993.

18. Hannon GJ, Casso D and Beach D: KAP: A dual specificity phosphatase that interacts with cyclin-dependent kinases. Proc Natl Acad Sci USA 91: 1731-1735, 1994.

19. Kawashima T, Hirose K, Satoh T, Kaneko A, Ikeda Y, Kaziro Y, Nosaka T and Kitamura T: MgcRacGAP is involved in the control of growth and differentiation of hematopoietic cells. Blood 96: 2116-2124, 2000.

20. Brabletz T: EMT and MET in metastasis: Where are the cancer stem cells? Cancer Cell 22: 699-701, 2012.

21. Mani SA, Guo W, Liao MJ, Eaton EN, Ayyanan A, Zhou AY, Brooks M, Reinhard F, Zhang CC, Shipitsin M, et al: The epithelial-mesenchymal transition generates cells with properties of stem cells. Cell 133: 704-715, 2008. 
22. Zou J, Li H, Huang Q, Liu X, Qi X, Wang Y, Lu L and Liu Z: Dopamine-induced SULT1A3/4 promotes EMT and cancer stemness in hepatocellular carcinoma. Tumour Biol 39: $1010428317719272,2017$.

23. Santamaria PG, Moreno-Bueno G, Portillo $F$ and Cano A: EMT: Present and future in clinical oncology. Mol Oncol 11: 718-738, 2017.

24. Nistico P: Bissell MJandRadisky DC: Epithelial-mesenchymal transition: general principles and pathological relevance with special emphasis on the role of matrix metalloproteinases. Cold Spring Harb Perspect Biol 4: a011908, 2012.
25. Gaianigo N: Melisi D and Carbone C: EMT and Treatment Resistance in Pancreatic Cancer. Cancers (Basel) 9: 122, 2017.

cc) (i) $\odot$ This work is licensed under a Creative Commons Attribution-NonCommercial-NoDerivatives 4.0 International (CC BY-NC-ND 4.0) License. 